January 19, 2021

\title{
PERIODS OF HILBERT MODULAR FORMS, KRONECKER SERIES AND COHOMOLOGY
}

\author{
YOUNGJU CHOIE
}

\begin{abstract}
Generalizing a result of [24, 8] about elliptic modular forms, we give a closed formula for the sum of all Hilbert Hecke eigenforms over a totally real number field with strict class number 1 , multiplied by their period polynomials, as a single product of the Kronecker series.
\end{abstract}

\section{Introduction}

Based on Bol's result [3] Eichler initiated a theory of the periods of integrals so that an automorphic form of the first or second kind leads to a cohomology class in the mapping of a Fuchsian group into a polynomial module and the (converse) correspondence of each such cohomology class leads to an automorphic form in one complex variable [9]. Shimura extended this theory by showing that the structure of an abelian variety in certain cases can be also given to the periods of such integrals and showed critical values of the $L$-functions attached to elliptic modular forms can be computed explicitly using the cohomology group [20]. This method was developed by Manin [16] who proved an algebraic theorem for the periods of elliptic cusp forms for the full modular group and studied $p$-adic properties of the algebraic factors in $L$ functions. Kohnen-Zagier [14] further extended this theory to elliptic modular forms including Eisenstein series [14] and studied forms whose period polynomials have arithmetically interesting rational structure relating to Bernoulli numbers, binary quadratic forms, zeta-functions of real quadratic fields, modular forms of half-integral weight and Hilbert modular forms. Hence, period

2000 Mathematics Subject Classification. 11F41, 11F50, 11F60, 11F67.

Key words and phrases. Hilbert modular form, parabolic cohomology, period polynomial, Kronecker series .

This work was partially supported by NRF 2018R1A4A1023590 and NRF 2017R1A2B2001807. 
polynomials, which allow us to compute the critical values of $L$-function of modular forms at once, give a rich source of relations between modular forms and arithmetic.

The period polynomial of an elliptic cusp form $f(\tau)=\sum_{\ell \geq 1} a_{f}(\ell) q^{\ell}(\tau \in$ $\mathbb{H}=$ upper half plane, $q=e^{2 \pi i \tau}$ ) of weight $k$ on $S L_{2}(\mathbb{Z})$ is the polynomial of degree $k-2$ defined by

$$
r_{f}(X)=\int_{0}^{i \infty} f(\tau)(\tau-X)^{k-2} d \tau
$$

or equivalently by

$$
r_{f}(X)=-\sum_{n=0}^{k-2} \frac{(k-2) !}{(k-2-n) !} \frac{L(f, n+1)}{(2 \pi i)^{n+1}} X^{k-2-n},
$$

where $L(f, s)=\sum_{n \geq 1} \frac{a_{f}(n)}{n^{s}}(R e(s) \gg 0)$. The maps $f \rightarrow r_{f}^{e v}$ and $f \rightarrow r_{f}^{o d}$ assigning to $f$ the even and odd parts of $r_{f}$ are both injective with known images from the Eichler-Shimura-Manin theory.

When $f$ is a Hecke eigenform then one has the two-variable polynomial

$$
r_{f}(X, Y):=\frac{r_{f}^{e v}(X) r_{f}^{o d}(Y)+r_{f}^{o d}(X) r_{f}^{e v}(Y)}{(2 i)^{k-3}<f, f>} \in \mathbb{Q}_{f}[X, Y]
$$

where $\mathbb{Q}_{f}$ is the field generated by Fourier coefficients of $f$ over $\mathbb{Q}$.

Zagier [24] found the following attractive formula:

$$
\begin{aligned}
& \frac{(X Y-1)(X+Y)}{X^{2} Y^{2}} T^{-2}+\sum_{k \geq 2} \sum_{f \in \mathcal{B}_{k}} r_{f}(X, Y) f(\tau) \frac{T^{k-2}}{(k-2) !} \\
= & F_{\tau}(T,-X Y T) F_{\tau}(X T, Y T), F_{\tau}(u, v)=\frac{\theta^{\prime}(0) \theta(u+v)}{\theta(u) \theta(v)}
\end{aligned}
$$

where $\theta(u)=\sum_{n \in \mathbb{Z}}(-1)^{n} q^{\frac{1}{2}\left(n+\frac{1}{2}\right)^{2}} e^{\left(n+\frac{1}{2}\right) u}$ is a Jacobi theta function and $\mathcal{B}_{k}$ is a set of all Hecke eigenforms of weight $k$ on $S L_{2}(\mathbb{Z})$.

The identity by Zagier (1.2) relates a generating function, which contains all Hecke eigenforms together with all critical values, to the Jacobi form $F_{\tau}(u, v)$. Such expansions with respect to the variable $T$ give an algorithm to compute 
Hecke eigenforms (see [24] for more details).

It took almost 30 years to see that such an identity (1.2) is not accidental but also exists for a general group $\Gamma_{0}(N)$ (see [8]). Now it seems natural to ask if one can get such a relation for general automorphic forms. In this paper we attempt to get such a formula, namely, an identity between a generating function of periods of Hilbert modular forms over totally real number fields with strict class number one and Jacobi forms (see Theorem 2.2). The function $F_{\tau}(u, v)$ in (1.2) was introduced by Kronecker in a more general form (see page 70 in [23]) and several properties of that have been explored by Zagier [24]. The essential property of $F_{\tau}(u, v)$ is that it can be identified as a sum of derivatives of Eisenstein series (called the "Kuznetsov lifting") and using this fact we are able to extend Zaiger' identity to that for a totally real number field. The main result of this paper shows the first connection between the Kronecker series and the critical $L$-values of Hilbert modular forms over the totally real number fields. It also gives a systematic way to compute Hilbert Hecke eigenforms and the special values of $L$-functions by taking the expansions of the Kronecker series.

This paper is organized as follows: in section 2 we state (Main) Theorem 2.2 after introducing necessary notations. In section 3 the analog of EisensteinKronecker series over a totally real number field and the rationality of period polynomials of Hilbert modular forms are discussed. Section 4 gives detailed proof of Main Theorem. Finally, we give a comment on a connection between parabolic cohomology and a period theory of Hilbert modular forms. Also, we discuss a possible application of the Kronecker series to evaluate the special $L$-values of a general automorphic form as a conclusion.

Acknowledgement I would like to thank the referees for numerous helpful comments and suggestions which greatly improved the exposition of this paper.

\section{Notations and statement of Main Theorem}

\subsection{Notation.}


- $\mathbb{F}$ : a totally real number field of degree $t$ with discriminant $D$ and strict class number 1

- $\mathcal{O}$ : the ring of integers of $\mathbb{F}$ containing a unit of negative norm

- $\mathcal{O}^{*}$ : the group of units of $\mathcal{O}$

- $\mathcal{O}^{*,+}$ : the group of totally positive units of $\mathcal{O}$

- $U^{+}=\left\{\epsilon^{2}: \epsilon \in \mathcal{O}^{*,+}\right\}$

- $\alpha \succ 0$ : a totally positive element

- $\alpha_{1} \rightarrow \alpha_{2}, \cdots, \alpha_{t}$ for the conjugation

- $\mathcal{N}(\alpha)=\prod_{j=1}^{t} \alpha_{j}$ the norm

- $\operatorname{tr}(\alpha)=\sum_{j=1}^{t} \alpha_{j}$ the trace

- $\mathfrak{D}$ : the different of $\mathbb{F}$

- $\zeta_{\mathbb{F}}(s)=\sum_{c \subset \mathcal{O}} \frac{1}{\mathcal{N}(c)^{s}}$, where $s \in \mathbb{C}$ and the integral ideal $c$

- $|\mathbf{r}|=\sum_{i=1}^{t} r_{i}, \mathbf{r}+\mathbf{r}^{\prime}=\left(r_{1}+r_{1}^{\prime}, \cdots, r_{t}+r_{t}^{\prime}\right), \Gamma(\mathbf{r}+\mathbf{1})=\mathbf{r} !=r_{1} ! \cdots r_{t} !,\left(\begin{array}{c}\mathbf{r} \\ \mathbf{r}^{\prime}\end{array}\right)=$ $\left(\begin{array}{l}r_{1} \\ r_{1}^{\prime}\end{array}\right)\left(\begin{array}{l}r_{2} \\ r_{2}^{\prime}\end{array}\right) \cdots\left(\begin{array}{l}r_{t}^{\prime} \\ r_{t}^{\prime}\end{array}\right)$ for $\mathbf{r}=\left(r_{1}, r_{2}, \cdots, r_{t}\right), \mathbf{r}^{\prime}=\left(r_{1}^{\prime}, r_{2}^{\prime}, \cdots, r_{t}^{\prime}\right) \in \mathbb{Z}_{\geq 0}^{t}$

- $z^{\mathbf{r}}=z_{1}^{{ }^{r_{1}}} z_{2}{ }^{r_{2}} \cdots z_{t}{ }^{r_{t}}, \operatorname{tr}(\mathbf{m} z)=\sum_{j=1}^{t} m_{j} z_{j}, \mathcal{N}(z)=\prod_{j=1}^{t} z_{i}$ for $z=$ $\left(z_{1}, z_{2}, \cdots, z_{t}\right) \in \mathbb{C}^{t}$ and $\mathbf{m} \in \mathbb{F}$

- $\mathbb{H}^{t}$ : the $t$-copies of complex upper half plane $\mathbb{H}$

- $\tau=\left(\tau_{1}, \cdots, \tau_{t}\right)=x+\sqrt{-1} y \in \mathbb{H}^{t}, x=\left(x_{1}, \cdots, x_{t}\right) \in \mathbb{R}^{t}, y=$ $\left(y_{1}, \cdots, y_{t}\right) \in\left(\mathbb{R}^{+}\right)^{t}, q=\prod_{j=1}^{t} q_{j}, q_{j}=e^{2 \pi i \tau_{j}}, 1 \leq j \leq t$.

- $\sigma=\left(\sigma_{1}, \cdots, \sigma_{t}\right) \in \Gamma=S L_{2}(\mathcal{O})^{t}$ : an element in the Hilbert modular group

- The action of the group $\Gamma$, which is embedded into $S L_{2}(\mathbb{R}) \times \cdots \times$ $S L_{2}(\mathbb{R})$, on $\mathbb{H}^{t}$ is given by linear fractional transformations

$$
\left(\begin{array}{ll}
a & b \\
c & d
\end{array}\right) \tau=\frac{a \tau+b}{c \tau+d}=\left(\frac{a_{1} \tau_{1}+b_{1}}{c_{1} \tau_{1}+d_{1}}, \cdots, \frac{a_{t} \tau_{t}+b_{t}}{c_{t} \tau_{t}+d_{t}}\right), \tau=\left(\tau_{1}, \cdots, \tau_{t}\right) \in \mathbb{H}^{t}
$$

- For a holomorphic function $\chi$ on $\mathbb{H}^{t}$,

$$
\chi^{(\ell)}(\tau)=\frac{\partial^{|\ell|}}{\partial \tau^{\ell}} \chi(\tau):=\frac{\partial^{|\ell|}}{\partial \tau_{1}^{\ell_{1}} \cdots \partial \tau_{t}^{\ell_{t}}} \chi(\tau), \quad \forall \ell=\left(\ell_{1}, \cdots, \ell_{t}\right) \in \mathbb{Z}_{\geq 0}^{t}
$$

- $\mathbb{D}^{\ell}(\chi(\tau)):=\chi^{(\ell)}(\tau)$

- $S_{\mathbf{k}} \subset M_{\mathbf{k}}$ : the space of Hilbert cusp form $\subset$ the space of Hilbert modular form on $\Gamma$ with a parallel weight $\mathbf{k}=(k, \cdots, k)$, even $k \geq 2$.

- $\mathcal{B}_{k}^{0} \subset \mathcal{B}_{k}$ : a basis, consisting of all normalized Hecke eigenforms, of $S_{\mathrm{k}} \subset M_{\mathrm{k}}$, respectively 
PERIODS OF HILBERT MODULAR FORMS, KRONECKER SERIES AND COHOMOLOGY5

- $\mathbb{Q}_{f}$ : the field spanned by Fourier coefficients of $f$ over $\mathbb{Q}$

- $\langle f, g\rangle:=\int_{\Gamma \backslash \mathbb{H}^{t}} f(\tau) \overline{g(\tau)} \frac{d x d y}{y^{2}}$, the Petersson inner product for $f \in$ $S_{\mathbf{k}}, g \in M_{\mathbf{k}}$

- For a function $f$ on $\mathbb{H}^{t}$ and $\ell=\left(\ell_{1}, \cdots, \ell_{t}\right) \in \mathbb{Z}^{t}$,

$$
\left(\left.f\right|_{\ell} \sigma\right)(z):=(c z+d)^{-\ell} f\left(\frac{a z+b}{c z+d}\right), \sigma=\left(\begin{array}{ll}
a & b \\
c & d
\end{array}\right) \in \Gamma
$$

2.2. Statement of Main Theorem. Take a cusp form $f(\tau)=\sum_{\mathcal{D}^{-1} \ni \nu \succ 0} a_{f}(\nu) e^{2 \pi i t r(\nu \tau)}$ in $S_{\mathbf{k}}$ and consider the complete $L$-function of $f:$ for $s \in \mathbb{C}$,

$$
\Lambda(f, s):=\int_{\mathbb{R}_{+}^{t} / U^{+}} f(i y) y^{s-1} d y=D^{s}(2 \pi)^{-t s} \Gamma(s)^{t} L(f, s),
$$

where $L(f, s)=\sum_{\mathcal{D}^{-1} / U^{\dagger} \ni \nu \succ 0} \frac{a_{f}(\nu)}{\mathcal{N}(\nu)^{s}}(\operatorname{Re}(s) \gg 0)$. It is well-known that $\Lambda(f, s)$ has an analytic continuation and functional equation [4, 11]

$$
\Lambda(f, s)=(-1)^{\frac{t k}{2}} \Lambda(f, k-s) .
$$

Consider the following polynomials in $X=\left(X_{1}, \cdots, X_{t}\right)$, called the even (odd) period polynomial associated to $f$ :

$$
\begin{aligned}
& R_{f}^{e v}(X):=\sum_{\substack{0 \leq n \leq k-2 \\
n \equiv 0(\bmod 2)}} \frac{\Gamma(k-1)^{t}}{\Gamma(n+1)^{t} \Gamma(k-n-1)^{t}} R_{k-2-n}(f) \mathcal{N}(X)^{n}, \\
& R_{f}^{\text {od }}(X):=\sum_{\substack{0<n<k-2 \\
n \equiv 1 \quad \bmod 2)}} \frac{(-1)^{n t} \Gamma(k-1)^{t}}{\Gamma(n+1)^{t} \Gamma(k-n-1)^{t}} R_{k-2-n}(f) \mathcal{N}(X)^{n}, \\
& R_{f}(X):=(-1)^{t}\left(R_{f}^{e v}(X)+R_{f}^{o d}(X)\right),
\end{aligned}
$$

where

$$
R_{n}(f):=\int_{\mathbb{R}_{+}^{t} / U^{+}} f(\tau) \tau^{n} d \tau=i^{t(n+1)} \Lambda(f, n+1) .
$$

Using the functional equation of $\Lambda(f, s)$ we get

$$
R_{k-2-n}(f)=(-1)^{t(n+1)} R_{n}(f)
$$

and so we get

$$
\mathcal{N}(X)^{k-2} R_{f}\left(-\frac{1}{X}\right)=(-1)^{t} R_{f}(X) .
$$


Let $f$ be a primitive (Hilbert) Hecke eigenform and consider the polynomial of the $2 t$-variables in $X=\left(X_{1}, \cdots, X_{t}\right)$ and $Y=\left(Y_{1}, \cdots, Y_{t}\right)$

$$
R_{f}(X, Y):=(-1)^{t} \frac{R_{f}^{e v}(X) R_{f}^{o d}(Y)+R_{f}^{e v}(Y) R_{f}^{o d}(X)}{D^{k-\frac{1}{2}}(2 i)^{t(k-3)}<f, f>} \in \mathbb{C}[X, Y] .
$$

It transforms under $\sigma \in \operatorname{Gal}(\mathbb{C} / \mathbb{Q})$ by $R_{\sigma(f)}=\sigma\left(R_{f}\right)$ so that $R_{f}(X, Y)$ has coefficients in the number field $\mathbb{Q}_{f}$ generated by the Fourier coefficients of $f$. Summing over the basis $\mathcal{B}_{k}^{0}$, consisting of all normalized Hecke eigenforms of $S_{\mathbf{k}}$, the following function

$$
C_{k}^{\text {cusp }}(X, Y ; \tau):=\sum_{f \in \mathcal{B}_{k}^{0}} R_{f}(X, Y) f(\tau)
$$

is in $\mathbb{Q}[[q]][X, Y]$ for each even integer $k \geq 2$. Further we extend the definition of $R_{f}(X, Y)$ (see section [3.2) to non-cusp forms and include the Eisenstein series in the sum (2.1). Then we define

$$
C_{k}(X, Y ; \tau):=\sum_{f \in \mathcal{B}_{k}} R_{f}(X, Y) f(\tau) .
$$

Example 2.1. Take $\mathbb{F}=\mathbb{Q}(\sqrt{5})$.

$$
\begin{aligned}
& C_{2}(X, Y ; \tau) \\
& =\sum_{f \in \mathcal{B}_{k}} R_{f}(X, Y) f(\tau)=2^{4} \cdot 3 \cdot 5 \frac{(\mathcal{N}(X)+\mathcal{N}(Y))(\mathcal{N}(X Y)+1)}{\mathcal{N}(X Y)} G_{\mathbb{F}, 2}(\tau),
\end{aligned}
$$

with the normalized Eisenstein series of weight $(k, k)$ on $\Gamma$ given by $G_{\mathbb{F}, k}(\tau)=\frac{\zeta_{\mathbb{F}}(1-k)}{2^{2}}+\sum_{\mathcal{D}^{-1} \ni \nu \succ 0} \sigma_{k-1}(\nu \mathcal{D}) e^{2 \pi i t r(\nu \tau)}, \sigma_{r}(\mathfrak{n})=\sum_{\mathfrak{c} \mid \mathfrak{n}} \mathcal{N}(\mathfrak{c})^{r}$.

(2) Let $\mathbb{F}=\mathbb{Q}(\sqrt{5})$ and take a unique cusp form $f$ of weight 8 on $\Gamma$. Using the example in [1] we get

$$
\frac{R_{f}^{e v}(X) R_{f}^{o d}(Y)}{5^{\frac{15}{2}}(2 i)^{10}<f, f>}=c\left(1+\frac{361}{2^{2} \cdot 5} X^{2}+\frac{361}{2^{2} \cdot 5} X^{4}+X^{6}\right)\left(Y+\frac{2}{3} Y^{3}+Y^{5}\right)
$$

up to rational constant multiple $c$. 
PERIODS OF HILBERT MODULAR FORMS, KRONECKER SERIES AND COHOMOLOGY7

Combining all these functions into a single generating function to define

$$
\begin{aligned}
& C(X, Y ; \tau ; T) \\
& :=\frac{(\mathcal{N}(X)+\mathcal{N}(Y))\left(\mathcal{N}(X Y)+(-1)^{t}\right)}{\mathcal{N}(X Y T)^{2}}+\sum_{k \geq 2} C_{k}(X, Y ; \tau) \frac{\mathcal{N}(T)^{k-2}}{\Gamma(k-1)^{t}}
\end{aligned}
$$

On the other hand, consider

$$
F_{\tau}(u, v):=(-2)^{t} \sum_{k \geq 0} \widetilde{G_{\mathbb{F}, k}}\left(\tau, \frac{u v}{2 \pi i}\right)\left(\mathcal{N}(u)^{k-1}+\mathcal{N}(v)^{k-1}\right), u, v \in \mathbb{C}^{t}
$$

where

$$
\widetilde{G_{\mathbb{F}, k}}(\tau, \lambda):=\left\{\begin{array}{cc}
\sum_{\ell=\left(\ell_{1}, \cdots, \ell_{t}\right) \in \mathbb{Z}_{\geq 0}^{t}} \frac{\lambda^{\ell}}{\ell !(\ell+\mathbf{k}-\mathbf{1}) !} \mathbb{D}^{\ell}\left(G_{\mathbb{F}, k}(\tau)\right) & \text { if } k \geq 2 \\
\frac{1}{2^{t}} & \text { if } k=0
\end{array}\right\}, \lambda \in \mathbb{C}^{t}
$$

and a normalized Hilbert Eisenstein series $G_{\mathbb{F}, k}(\tau)$ (p 20 in [11]) defined by

$$
\begin{aligned}
E_{\mathbb{F}, k}(\tau) & :=\frac{D^{\frac{1}{2}-k}(2 \pi i)^{t k}}{\Gamma(k)^{t}}\left(\frac{1}{2^{t}} \zeta_{\mathbb{F}}(1-k)+\sum_{\substack{\nu \in \mathfrak{D}^{-1} \\
\nu \succ 0}} \sigma_{k-1}(\nu \mathfrak{D}) e^{2 \pi i t r(\nu \tau)}\right) \\
& :=\frac{D^{\frac{1}{2}-k}(2 \pi i)^{t k}}{\Gamma(k)^{t}} G_{\mathbb{F}, k}(\tau), \sigma_{r}(\mathfrak{n})=\sum_{\mathfrak{c} \mid \mathfrak{n}} \mathcal{N}(\mathfrak{c})^{r} .
\end{aligned}
$$

Now we state the main result of this paper :

Theorem 2.2. (Main Theorem) Let $C(X, Y ; \tau ; T)$ be the generating function of the periods of Hilbert modular forms given in (2.3). Then we have

(1) $C(X, Y ; \tau ; T) \in \frac{1}{\mathcal{N}(X Y T)^{2}} \mathbb{Q}[X, Y][[q, T]]$.

(2) $C(X, Y ; \tau ; T)=F_{\tau}(T,-X Y T) F_{\tau}(X T, Y T)$.

Remark 2.1. $\widetilde{G_{\mathbb{F}, k}}(\tau, \lambda)$ in $(2.5)$ is the "Kuznetsov lifting" of the Hilbert Eisenstein series $G_{\mathbb{F}, \mathbf{k}}(\tau)$. Its modular transformation property is known (see Theorem 2 in [6]) : for any $\left(\begin{array}{ll}a & b \\ c & d\end{array}\right) \in \Gamma, k \geq 2$,

$$
\mathcal{N}(c \tau+d)^{-k} e^{-\operatorname{tr}\left(\frac{c \lambda}{c \tau+d}\right)} \widetilde{G_{\mathbb{F}, k}}\left(\frac{a \tau+b}{c \tau+d}, \frac{\lambda}{c \tau+d}\right)=\widetilde{G_{\mathbb{F}, k}}(\tau, \lambda)
$$


and its generating function $\mathcal{F}_{\tau}(u, v):=(-2)^{t} \sum_{k \geq 2} \widetilde{G_{\mathbb{F}, k}}\left(\tau, \frac{u v}{2 \pi i}\right)\left(\mathcal{N}(u)^{k-1}+\right.$ $\left.\mathcal{N}(v)^{k-1}\right)$ behaves as a Jacobi-like form [10, 7] with a modular transformation property

$$
\mathcal{F}_{\frac{a \tau+b}{c \tau+d}}\left(\frac{u}{c \tau+d}, \frac{v}{c \tau+d}\right)=\mathcal{N}(c \tau+d) e^{\operatorname{tr}\left(\frac{c u v}{c \tau+d}\right)} \mathcal{F}_{\tau}(u, v), \forall\left(\begin{array}{cc}
a & b \\
c & d
\end{array}\right) \in \Gamma .
$$

\section{Algebraicity and Period of Hecke eigen forms}

3.1. Algebraicity. The study of period relation for automorphic forms was started by Shimura. He showed the existence of relations up to factors in $\overline{\mathbb{Q}}^{*}$ in many instances and made a general conjecture relating periods of Hilbert modular varieties and their compact analogs, that is, the quaternionic modular varieties [17, 18]. There is a weaker conjecture, which gives a relation between a product of two periods, called the quadratic periods, may be interpreted, up to algebraic factors, as Petersson inner products. This was proved by M. Harris [12] under a certain technical condition. More precisely, for each $m, 0 \leq m \leq k-2, \Lambda(f, m+1)$ is called the critical values.

Theorem 3.1. (Theorem 4.3 in [19]) Let $f$ be a Hilbert Hecke eigenform of weight $\mathbf{k}=(k, \cdots, k)$ over a totally real number field $\mathbb{F}$ of degree $t$ and $\sigma \in \operatorname{Gal}(\overline{\mathbb{Q}} / \mathbb{Q})$.

(1) For each $r \in \mathbb{Z}^{t} / 2 \mathbb{Z}^{t}$ and for $f^{\sigma}, \sigma \in \operatorname{Gal}(\overline{\mathbb{Q}} / \mathbb{Q})$, there exist nonzero complex numbers $\omega_{f}^{r}$ such that $\left(\frac{L(f, m)}{(2 \pi i)^{t m} \omega_{f}^{r}}\right)^{\sigma}=\frac{L\left(f^{\sigma}, m\right)}{(2 \pi i)^{t m} \omega_{f \sigma}^{r}}$, for any integer $m$ such that $0<m<k$.

(2) $\frac{L(f, m)}{(2 \pi i)^{t m} \omega_{f}^{r}} \in \mathbb{Q}_{f}$.

(3) If $p=\left(p_{1}, \cdots, p_{t}\right), r=\left(r_{1}, \cdots, r_{t}\right)$ with $p_{i}+r_{i} \equiv 1(\bmod 2), 1 \leq \forall i \leq t$, we have $\frac{w_{f}^{p} \cdot w_{f}^{r}}{\langle f, f\rangle} \in \mathbb{Q}_{f}$ and $\left(\frac{w_{f}^{p} \cdot w_{f}^{r}}{\langle f, f\rangle}\right)^{\sigma}=\frac{w_{f f}^{p} \cdot w_{f}^{r}}{\left\langle f^{\sigma}, f^{\sigma}\right\rangle}$.

3.2. Period of non-cusp forms. Since $\mathcal{B}_{k}$ in (2.2) contains non-cusp forms one needs to explain "period function" corresponding to a non-cusp form $f$. Take a non-cusp form $f(\tau)=\sum_{0 \preceq \nu \in \mathfrak{D}^{-1}} a_{f}(\nu) e^{2 \pi i t r(\nu \tau)}$ in $M_{\mathbf{k}}$ and consider

$$
\Lambda(f, s):=\int_{\left(\mathbb{R}_{+}\right)^{t} / U^{+}}\left(f(i y)-a_{f}(0)\right) y^{s-1} d y=D^{s}(2 \pi)^{-t s} \Gamma(s)^{t} L(f, s), s \in \mathbb{C} .
$$


PERIODS OF HILBERT MODULAR FORMS, KRONECKER SERIES AND COHOMOLOGY9

It has a meromorphic continuation to $\mathbb{C}$ and satisfies a functional equation $\Lambda(f, s)=(-1)^{\frac{t k}{2}} \Lambda(f, k-s)$, but now has simple poles of residue as $-a_{f}(0)$ and $(-1)^{k t} a_{f}(0)$, up to a constant multiple, at $s=0$ and $s=k$, respectively. Define

$$
\begin{gathered}
R_{f}(X):=\frac{(-1)^{t} \sqrt{D} \cdot a_{f}(0)}{(k-1)^{t}}\left(\mathcal{N}(X)^{k-1}+(-1)^{t} \mathcal{N}(X)^{-1}\right) \\
+\sum_{n=0}^{k-2}(-1)^{\frac{t(k+n-1)}{2}} \frac{\Gamma(k-1)^{t}}{\Gamma(n+1)^{t} \Gamma(k-n-1)^{t}} \Lambda(f, k-1-n) \mathcal{N}(X)^{n} .
\end{gathered}
$$

The assumption that $\mathbb{F}$ has the strict class number 1 implies that the space of Hilbert modular forms is a direct sum (see [4], p 12)

$$
M_{\mathbf{k}}=S_{\mathbf{k}} \oplus<G_{\mathbb{F}, k}>
$$

and, so (2.2) becomes

$$
C_{k}(X, Y ; \tau)=\sum_{f \in \mathcal{B}_{k}^{0}} R_{f}(X, Y) f(\tau)+R_{G_{\mathbb{F}, k}}(X, Y) G_{\mathbb{F}, k}(\tau),
$$

where $R_{G_{\mathbb{F}, k}}(X, Y)$, defined by

$$
R_{G_{\mathbb{F}, k}}(X, Y)=(-1)^{t} \frac{R_{G_{\mathbb{F}, k}}^{e v}(X) R_{G_{\mathbb{F}, k}}^{o d}(Y)+R_{G_{\mathbb{F}, k}}^{e v}(Y) R_{G_{\mathbb{F}, k}}^{o d}(X)}{D^{k-\frac{1}{2}}(2 i)^{t(k-3)}<G_{\mathbb{F}, k}, G_{\mathbb{F}, k}>},
$$

is a symmetrized sum of the product of period polynomials of the normalized Hecke Eisenstein series $G_{\mathbb{F}, k}(\tau)$ given as followings:

Proposition 3.2. Take

$$
w_{G_{\mathbb{F}, k}}^{-}=\frac{\sqrt{D} \Gamma(k-1)^{t}}{2^{t}}, w_{G_{\mathbb{F}, k}}^{+}=\frac{D^{k-\frac{3}{2}} \zeta_{\mathbb{F}}(k-1)}{(2 \pi i)^{t(k-1)}} w_{G_{\mathbb{F}, k}}^{-}
$$

and

$$
\begin{aligned}
& p_{k}^{+}(X)=\mathcal{N}(X)^{k-2}+(-1)^{t}, \\
& p_{k}^{-}(X)=\sum_{-1 \leq n \leq k-1, n \equiv 1} \frac{\zeta_{\mathbb{F}}(1-(n+1)) \zeta_{\mathbb{F}}(n+2-k)}{\Gamma(n+1)^{t} \Gamma(k-n-1)^{t}} \mathcal{N}(X)^{n}
\end{aligned}
$$

For $k \geq 2$ the period function of $G_{\mathbb{F}, k}(\tau)$ is given by

$$
R_{G_{\mathbb{F}, k}}(X)=(-1)^{t}\left(w_{G_{\mathbb{F}, k}}^{-} \cdot p_{k}^{-}(X)+w_{G_{\mathbb{F}, k}}^{+} \cdot p_{k}^{+}(X)\right)
$$


so that

$$
R_{G_{\mathbb{F}, k}}^{e v}(X)=w_{G_{\mathbb{F}, k}}^{+} \cdot p_{k}^{+}(X) \text { and } R_{G_{\mathbb{F}, k}}^{o d}(X)=w_{G_{\mathbb{F}, k}}^{-} \cdot p_{k}^{-}(X) .
$$

Remark 3.3. (1) Like in the case of an elliptic modular form (see [14]), the period function $R_{G_{\mathbb{F}, k}}(X)$ is $\frac{1}{\mathcal{N}(X)}$ times a polynomial :

$$
R_{G_{\mathbb{R}, k}}(X) \in \frac{1}{\mathcal{N}(X)} \mathbb{C}[X]
$$

(2) [11] Note that $\frac{D^{n-\frac{1}{2}} \zeta_{\mathbb{F}}(n) \Gamma(n)^{t}}{(2 \pi i)^{t n}}=\frac{\zeta_{\mathbb{F}}(1-n)}{2^{t}}$ and $\zeta_{\mathbb{F}}(-n)=0$ for any positive even integer $n$.

(3) (a) [26] Let $\mathbb{F}$ be a real quadratic field with discriminant $D$. It is known that $\zeta_{\mathbb{F}}(1-n)=B_{n} B_{n, \chi}$ for even positive integer $n$. $B_{r}$ and $B_{r, \chi}$ are the $r$ th Bernoulli number $\left(B_{0}=1, B_{1}=-\frac{1}{2}, B_{2}=\frac{1}{6}, \cdots\right)$ and the $r$ th twisted Bernoulli number $\left(B_{1, \chi}=\frac{1}{D} \sum_{a=1}^{D} \chi(a) a, B_{2, \chi}=\right.$ $\left.\frac{1}{D} \sum_{a=1}^{D} \chi(a) a^{2}-\sum_{a=1}^{D} \chi(a) a, B_{3, \chi}=\cdots\right)$, respectively. Here $\chi$ $(\bmod D)$ is a primitive character defined by $\chi(\cdot)=\left(\frac{D}{.}\right)$.

(b) (open problem) It is well known that the generating functions of $B_{j}$ and $B_{j, \chi}$ are

$$
\sum_{n=0}^{\infty} B_{n} \frac{t^{n}}{n !}=\frac{t e^{t}}{e^{t}-1} \text { and } \sum_{m \geq 0} B_{m, \chi} \frac{t^{m}}{m !}=\sum_{j=1}^{D} \frac{\chi(j) t e^{j t}}{e^{D t}-1}
$$

Similarly, it will be interesting to express the following generating function

$$
\sum_{m \geq 2}^{\infty} B_{m} B_{m, \chi} \frac{t^{m}}{m !}=\sum_{m \geq 2}^{\infty} \zeta_{\mathbb{F}}(1-m) \frac{t^{m}}{m !},
$$

as elementary functions.

Proof of Proposition 3.2: The period polynomial of $G_{\mathbb{F}, k}(\tau)$ can be computed from the definition in (3.1) : using $\Lambda\left(G_{\mathbb{F}, k}, n+1\right)=\frac{D^{n+1} \Gamma(n+1)^{t}}{(2 \pi)^{t(n+1)}} \zeta_{\mathbb{F}}(n+1) \zeta_{\mathbb{F}}(n+$ 
PERIODS OF HILBERT MODULAR FORMS, KRONECKER SERIES AND COHOMOLOGY1

$2-k)$, we have

$$
\begin{aligned}
& R_{G_{\mathbb{F}, k}}(X)=\frac{(-1)^{t} \sqrt{D} \zeta_{\mathbb{F}}(1-k)}{2^{t}(k-1)^{t}}\left(\mathcal{N}(X)^{k-1}+(-1)^{t} \mathcal{N}(X)^{-1}\right) \\
& +\frac{(-1)^{t} D^{k-1} \Gamma(k-1)^{t} \zeta_{\mathbb{F}}(k-1)}{2^{t}(2 \pi i)^{t(k-1)}}\left(\mathcal{N}(X)^{k-2}+(-1)^{t}\right) \\
& +\sum_{n=1}^{k-3}(-1)^{\frac{t(k+n-1)}{2}} \frac{\Gamma(k-1)^{t} D^{k-n-1}}{(2 \pi)^{t(k-n-1)} \Gamma(n+1)^{t}} \zeta_{\mathbb{F}}(1-(n+1)) \zeta_{\mathbb{F}}(k-1-n) \mathcal{N}(X)^{n}
\end{aligned}
$$

(using the functional equation of $\zeta_{\mathbb{F}}(s)$ of Remark 3.3-(2))

$$
\begin{aligned}
& =\frac{(-1)^{t} D^{k-1} \Gamma(k-1)^{t} \zeta_{\mathbb{F}}(k-1)}{2^{t}(2 \pi i)^{t(k-1)}}\left(\mathcal{N}(X)^{k-2}+(-1)^{t}\right) \\
& +\frac{(-1)^{t} \sqrt{D} \Gamma(k-1)^{t}}{2^{t}} \sum_{-1 \leq n \equiv 1} \sum_{(\bmod 2) \leq k-1} \frac{\zeta_{\mathbb{F}}(1-(n+1)) \zeta_{\mathbb{F}}(n+2-k)}{\Gamma(n+1)^{t} \Gamma(k-n-1)^{t}} \mathcal{N}(X)^{n} \\
& =(-1)^{t}\left(\omega_{G_{\mathbb{F}, k}}^{+} p_{k}^{+}(X)+\omega_{G_{\mathbb{F}, k}}^{-} p_{k}^{-}(X)\right) .
\end{aligned}
$$

This completes a proof.

3.2.1. Petersson scalar product of Eisenstein Series. The Petersson scalar product of a $S L_{2}(\mathbb{Z})$-invariant function had been defined by Zagier [25] using Rankin-Selberg method. Similarly we have

\section{Proposition 3.4.}

$$
<G_{\mathbb{F}, k}(\tau), G_{\mathbb{F}, k}(\tau)>=\frac{\Gamma(k-1)^{t} \zeta_{\mathbb{F}}(k-1)}{(4 \pi)^{t(k-1)}} \frac{\zeta_{\mathbb{F}}(1-k)}{2^{t}}
$$

Proof of Proposition 3.4: Following the method in [25] the Petersson norm of the Hilbert Eisenstein series $G_{\mathbb{F}, k}(\tau)$ can be computed as

$$
<G_{\mathbb{F}, k}(\tau), G_{\mathbb{F}, k}(\tau)>=(-1)^{\frac{t k}{2}}(4 \pi)^{-t k} \Gamma(k)^{t} \cdot \zeta_{\mathbb{F}}^{*}(k) \zeta_{\mathbb{F}}^{*}(2-k)
$$

where $\zeta_{\mathbb{F}}^{*}(s):=D^{\frac{s}{2}} \pi^{-\frac{t s}{2}} \Gamma\left(\frac{s}{2}\right)^{t} \zeta_{\mathbb{F}}(s)=\zeta_{\mathbb{F}}^{*}(1-s)$ (see p 57 [26]). Using the identities $\Gamma\left(\frac{k}{2}\right) \Gamma\left(\frac{k-1}{2}\right)=\Gamma(k-1) \sqrt{\pi} 2^{-(k-2)}$ and $\zeta_{\mathbb{F}}(k)=D^{-k+\frac{1}{2}} \frac{(2 \pi i)^{t k}}{2^{t} \Gamma(k)^{t}} \zeta_{\mathbb{F}}(1-k)$ we get the Petersson norm of the Eisenstein series $G_{\mathbb{F}, k}$. 
Now write the function $C_{k}(X, Y ; \tau)$ in (2.2) as a sum of $C_{k}^{c u s p}(X, Y ; \tau)$ in (2.1) and $C_{k}^{E i s}(X, Y ; \tau):=R_{G_{\mathbb{F}, k}}(X, Y) G_{\mathbb{F}, k}(\tau)$ :

$$
C_{k}(X, Y ; \tau)=C_{k}^{\text {cusp }}(X, Y ; \tau)+C_{k}^{E i s}(X, Y ; \tau)
$$

Proposition 3.5. We have

(1) $C_{k}^{E i s}(X, Y ; \tau)=(-1)^{t} \frac{2^{t} \Gamma(k-1)^{t}}{\zeta_{\mathbb{F}}(1-k)}\left(p_{k}^{+}(X) p_{k}^{-}(Y)+p_{k}^{+}(Y) p_{k}^{-}(X)\right) G_{\mathbb{F}, k}(\tau)$.

(2) $C(X, Y ; \tau ; T)=F_{\tau}(X T, Y T) F_{\tau}(T,-X Y T)$ as $\tau \rightarrow(i \infty, \cdots, i \infty)$

Proof of Proposition 3.5:

(1) From (3.2) recall that

$$
R_{G_{\mathbb{F}, k}}(X, Y)=(-1)^{t} \frac{R_{G_{\mathbb{F}, k}}^{e v}(X) R_{G_{\mathbb{F}, k}}^{o d}(Y)+R_{G_{\mathbb{F}, k}}^{e v}(Y) R_{G_{\mathbb{F}, k}}^{o d}(X)}{D^{k-\frac{1}{2}}(2 i)^{t(k-3)}<G_{\mathbb{F}, k}, G_{\mathbb{F}, k}>} .
$$

So, Proposition 3.2 and Proposition 3.4 imply that

$$
\begin{aligned}
& C_{k}^{E i s}(X, Y ; \tau)=R_{G_{\mathbb{F}, k}}(X, Y) G_{\mathbb{F}, k}(\tau) \\
& =\frac{\omega_{G_{\mathbb{F}, k}}^{+} \omega_{G_{\mathbb{R}, k}}^{-}\left(p_{k}^{+}(X) p_{k}^{-}(Y)+p_{k}^{+}(Y) p_{k}^{-}(X)\right)}{D^{k-\frac{1}{2}}(2 i)^{t(k-3)}<G_{\mathbb{F}, k}, G_{\mathbb{F}, k}>} G_{\mathbb{F}, k}(\tau) \\
& =(-1)^{t} \frac{2^{t} \Gamma(k-1)^{t}}{\zeta_{\mathbb{F}}(1-k)}\left(p_{k}^{+}(X) p_{k}^{-}(Y)+p_{k}^{+}(Y) p_{k}^{-}(X)\right) G_{\mathbb{F}, k}(\tau) .
\end{aligned}
$$

(2) Using Proposition 3.5 part (1) the value of $C(X, Y ; \tau ; T)$ as $\tau \rightarrow$ $(i \infty, \cdots, i \infty)$ is

$$
\begin{aligned}
& C(X, Y ;(i \infty, \cdots, i \infty) ; T) \\
= & \frac{(\mathcal{N}(X)+\mathcal{N}(Y))\left(\mathcal{N}(X Y)+(-1)^{t}\right)}{\mathcal{N}(X Y T)^{2}}+\sum_{k \geq 2} C_{k}^{E i s}(X, Y ;(i \infty, \cdots, i \infty)) \frac{\mathcal{N}(T)^{k-2}}{\Gamma(k-1)^{t}} \\
= & \frac{(\mathcal{N}(X)+\mathcal{N}(Y))\left(\mathcal{N}(X Y)+(-1)^{t}\right)}{\mathcal{N}(X Y T)^{2}}+(-1)^{t} \sum_{k \geq 2}\left(p_{k}^{+}(X) p_{k}^{-}(Y)+p_{k}^{+}(Y) p_{k}^{-}(X)\right) \mathcal{N}(T)^{k-2}
\end{aligned}
$$


PERIODS OF HILBERT MODULAR FORMS, KRONECKER SERIES AND COHOMOLOGY3

since $G_{\mathbb{F}, k}(i \infty)=\frac{\zeta_{\mathbb{F}}(1-k)}{2^{t}}$. On the other hand, a direct computation shows that

$$
\begin{aligned}
& \left.F_{\tau}(T,-X Y T) F_{\tau}(X T, Y T)\right|_{\tau \rightarrow(i \infty, \cdots, i \infty)} \\
& =\frac{(\mathcal{N}(X)+\mathcal{N}(Y))\left(\mathcal{N}(X Y)+(-1)^{t}\right)}{\mathcal{N}(X Y T)^{2}}+(-1)^{t} \sum_{k \geq 2}\left(p_{k}^{+}(X) p_{k}^{-}(Y)+p_{k}^{+}(Y) p_{k}^{-}(X)\right) \mathcal{N}(T)^{k-2}
\end{aligned}
$$

This completes the proof of Proposition 3.5.

\section{ProOFs}

\subsection{Proof of Theorem 2.2 (Main Theorem). (1) Using Theorem}

3.1 with a proper choice of the Petersson norm $\langle f, f\rangle$, we see that

$$
R_{f}(X, Y):=(-1)^{t} \frac{R_{f}^{e v}(X) R_{f}^{o d}(Y)+R_{f}^{e v}(Y) R_{f}^{o d}(X)}{D^{k-\frac{1}{2}}(2 i)^{t(k-3)}<f, f>} \in \mathbb{Q}_{f}[X, Y], f \in S_{\mathbf{k}} .
$$

With an action of $\sigma \in \operatorname{Gal}\left(\mathbb{C} / \mathbb{Q}_{f}\right)$ by $R_{\sigma(f)}=\sigma\left(R_{f}\right)$ we see that

$$
\begin{gathered}
C(X, Y ; \tau ; T)=\frac{(\mathcal{N}(X)+\mathcal{N}(Y))\left(\mathcal{N}(X Y)+(-1)^{t}\right)}{\mathcal{N}(X Y T)^{2}} \\
+\sum_{k \geq 2} \sum_{f \in \mathcal{B}_{k}} R_{f}(X, Y) f(\tau) \frac{\mathcal{N}(T)^{k-2}}{\Gamma(k-1)^{2}} \in \frac{1}{\mathcal{N}(X Y T)^{2}} \mathbb{Q}[X, Y][[q, T]] .
\end{gathered}
$$

This proves rationality of $C(X, Y ; \tau ; T)$.

(2) To prove Theorem 2.2 part (2) write the Taylor expansion (2.4)

$$
\begin{aligned}
F_{\tau}(u, v) & =\frac{1}{\mathcal{N}(u)}+\frac{1}{\mathcal{N}(v)} \\
& +(-2)^{t} \sum_{k \geq 2} \sum_{\ell \in \mathbb{Z}_{\geq 0}^{t}} \frac{\mathbb{D}^{\ell}\left(G_{\mathbb{F}, k}(\tau)\right)}{(2 \pi i)^{\ell} \ell !(\ell+\mathbf{k}-\mathbf{1}) !}\left(u^{\ell} v^{\ell+\mathbf{k}-1}+u^{\ell+\mathbf{k}-1} v^{\ell}\right)
\end{aligned}
$$

or write it as

$$
F_{\tau}(u, v)=\sum_{\mathbf{h}=(h, \cdots, h), \ell=\left(\ell_{1}, \cdots, \ell_{2}\right)} g_{h, \ell}(\tau)\left(u^{\ell} v^{\ell+\mathbf{h}-\mathbf{1}}+u^{\ell+\mathbf{h}-\mathbf{1}} v^{\ell}\right)
$$


with

$$
g_{h, \ell}(\tau)=\left\{\begin{array}{cc}
\frac{(-2)^{t}}{(2 \pi i)^{\ell} \Gamma(\ell+\mathbf{1}) \Gamma(\ell+\mathbf{h})} \mathbb{D}^{\ell}\left(G_{\mathbb{F}, h}(\tau)\right), & \text { if } h \geq 2, \ell_{i} \geq 0, i=1,2 \\
\frac{1}{2^{t}}, & \text { if } h=0, \ell=(0, \cdots, 0) \\
0, & \text { otherwise }
\end{array}\right\}
$$

Next let

$$
F_{\tau}(T,-X Y T) F_{\tau}(X T, Y T)=\sum_{k \geq 0} b_{\mathbf{k}}(X, Y ; \tau) \frac{\mathcal{N}(T)^{k-2}}{\Gamma(k-1)^{2}}
$$

Since we have already checked that

$$
C(X, Y ;(i \infty, \cdots, i \infty), T)=\left.F_{\tau}(T,-X Y T) F_{\tau}(X T, Y T)\right|_{\tau \rightarrow(i \infty, \cdots, i \infty)}
$$

in Proposition 3.5, it is enough to confirm that

$$
\frac{\left\langle b_{\mathbf{k}}(X, Y ; \cdot), f(\cdot)\right\rangle}{\langle f, f\rangle}=R_{f}(X, Y) \text { for each } f \in \mathcal{B}_{k}^{0} .
$$

From the expression (4.1) we see

$$
\begin{array}{ll}
b_{\mathbf{k}}(X, Y ; \tau)= & \sum_{\substack{\ell, \mathbf{h}, \ell^{\prime}, \mathbf{h}^{\prime} \\
\mathbf{h}+\mathbf{h}^{\prime}+2\left(\ell+\ell^{\prime}\right)=\mathbf{k} \\
\mathbf{h}=(h, \cdots, h), \mathbf{h}^{\prime}=\left(h^{\prime}, \cdots, h^{\prime}\right)}} g_{h, \ell}(\tau) g_{h^{\prime}, \ell^{\prime}}(\tau) \\
& \times\left[(-X Y)^{\ell+\mathbf{h}-\mathbf{1}}+(-X Y)^{\ell}\right]\left[X^{\ell^{\prime}} Y^{\ell^{\prime}+\mathbf{h}^{\prime}-\mathbf{1}}+X^{\ell^{\prime}+\mathbf{h}^{\prime}-\mathbf{1}} Y^{\ell^{\prime}}\right]
\end{array}
$$

The coefficients of $\mathcal{N}(X)^{p} \mathcal{N}(Y)^{q}$ with $q$ or $p$ equal to -1 or to $k-1$ involve only $G_{\mathbb{F}, k}(\tau)$ and have already been treated in Proposition 3.5. Also the coefficients of $\mathcal{N}(X)^{p} \mathcal{N}(Y)^{q}$ in (4.2) is invariant under $q \leftrightarrow k-2-p$ and $q \leftrightarrow p$ so that we may assume $0 \leq p<q \leq \frac{k-2}{2}$. For such $p, q$ the coefficient of 
PERIODS OF HILBERT MODULAR FORMS, KRONECKER SERIES AND COHOMOLOGY5

$\mathcal{N}(X)^{p} \mathcal{N}(Y)^{q}$ in (4.2) equals

$$
\begin{gathered}
\sum_{\begin{array}{c}
\ell, \ell^{\prime} \succeq-\mathbf{1} \\
\ell+\ell^{\prime}=\mathbf{p}=(p, \cdots, p)
\end{array}}(-1)^{|\ell|} g_{k-p-q-1, \ell}(\tau) g_{q-p+1, \ell^{\prime}}(\tau) \\
=\quad \sum_{\substack{\ell, \ell^{\prime} \succeq-\mathbf{1} \\
\ell+\ell^{\prime}=\mathbf{p}=(p, \cdots, p)}} 2^{2 t} \frac{(-1)^{|\ell|} \mathbb{D}^{\ell}\left(G_{\mathbb{F}, k-p-q-1}\right) \mathbb{D}^{\ell^{\prime}}\left(G_{\mathbb{F}, q-p+1}\right)}{\ell !(\ell+\mathbf{k}-\mathbf{p}-\mathbf{q}-2) ! \ell^{\prime} !\left(\mathbf{q}-\ell^{\prime}\right) !} \\
=\frac{2^{2 t}}{\Gamma(q+1)^{t} \Gamma(k-q-1)^{t}}\left[G_{\mathbb{F}, q-p+1}, G_{\mathbb{F}, k-q-p-1}\right]_{\mathbf{p}}^{H i l} .
\end{gathered}
$$

Here, $[\cdot \cdot]_{\mathbf{p}}^{\text {Hil }}$ denotes the $\mathbf{p}=(p, \cdots, p)$ th Rankin-Cohen bracket (see Corollary 1 in [6]) defined by

$$
\begin{aligned}
& {\left[G_{\mathbb{F}, q-p+1}, G_{\mathbb{F}, k-q-p-1}\right]_{\mathbf{p}}^{H i l}:=} \\
& \frac{1}{(2 \pi i)^{t p}} \sum_{0 \leq \ell_{i} \leq p} \frac{(-1)^{|\ell|} \Gamma(q+1)^{t} \Gamma(k-q-1)^{t}}{\ell^{\prime} !\left(\mathbf{k}+\ell^{\prime}-\mathbf{q}-\mathbf{p}-\mathbf{2}\right) ! \ell !(\mathbf{q}-\ell) !} \mathbb{D}^{\ell}\left(G_{\mathbb{F}, q-p+1}\right) \mathbb{D}^{\ell^{\prime}}\left(G_{\mathbb{F}, k-q-p-1}\right) . \\
& \ell=\left(\ell_{1}, \cdots, \ell_{t}\right) \\
& \ell+\ell^{\prime}=\mathbf{p}
\end{aligned}
$$

On the other hand we recall the following result (Theorem 3 in [6]) :

Theorem 4.1. [6] Suppose that $f(\tau)=\sum_{\mathcal{D}^{-1} \ni \nu \succ 0} a_{f}(\nu) e^{2 \pi i t r(\nu \tau)} \in S_{\mathbf{k}}$ and $g(\tau)=\sum_{\mathcal{D}^{-1} \ni \nu \succeq 0} a_{g}(\nu) e^{2 \pi i t r(\nu \tau)} \in M_{\mathbf{k}_{2}}$ with $k=k_{1}+k_{2}+2 p>2$. Then

$$
\frac{D^{\frac{1}{2}-k_{1}}(2 \pi i)^{t k_{1}}}{\Gamma\left(k_{1}\right)^{t}}<f,\left[G_{\mathbb{F}, k_{1}}, g_{k_{2}}\right]_{\mathbf{p}}>=\frac{\Gamma(k-1)^{t} \Gamma\left(k_{1}+p\right)^{t}}{(4 \pi)^{t(k-1)} \Gamma\left(k_{1}\right)^{t} \Gamma(p+1)^{t}} \sum_{\nu \succ 0} \frac{a_{f}(\nu) \overline{a_{g}(\nu)}}{\mathcal{N}(\nu)^{k-p-1}}
$$

Taking $g_{k_{2}}(\tau)=G_{\mathbb{F}, k}(\tau)$ in Theorem 4.1 we get

$$
\begin{aligned}
& <f,\left[G_{\mathbb{F}, k_{1}}, G_{\mathbb{F}, k_{2}}\right]_{\mathbf{p}}>=(-1)^{\frac{t k_{1}}{2}} \frac{D^{k_{1}-\frac{1}{2}} \Gamma(k-1)^{2} \Gamma\left(k_{1}+p\right)^{t}}{2^{t(k-1)}(2 \pi)^{t\left(k+k_{1}-1\right)} \Gamma(p+1)^{t}} \sum_{\nu>0} \frac{a_{f}(\nu) \sigma_{k_{2}-1}(\nu)}{\mathcal{N}(\nu)^{k-p-1}} \\
& =(-1)^{\frac{t k_{1}}{2}} \frac{D^{k_{1}-\frac{1}{2}} \Gamma(k-1)^{t} \Gamma\left(k_{1}+p\right)^{t}}{2^{t(k-1)}(2 \pi)^{t\left(k+k_{1}-1\right)} \Gamma(p+1)^{t}} L(f, k-p-1) L\left(f, k_{1}+p\right) \\
& \left(\text { since } R_{n}(f)=i^{t(n+1)} D^{n+1}(2 \pi)^{-t(n+1)} \Gamma(n+1)^{t} L(f, n+1)\right) \\
& =\frac{(-1)^{\frac{t(k-1)}{2}} \Gamma(k-1)^{t}}{D^{k-\frac{1}{2}} 2^{t(k-1)} \Gamma(p+1)^{t} \Gamma(k-p-1)^{t}} R_{k-p-2}(f) R_{k_{1}+p-1}(f) .
\end{aligned}
$$


And so we have that

$\sum_{f \in \mathcal{B}_{k}^{0}} \frac{R_{k-p-2}(f) R_{k_{1}+p-1}(f) f(\tau)}{i^{t(k-1)} D^{k-\frac{1}{2}} 2^{t(k-3)}<f, f>}=\frac{2^{2 t} \Gamma(p+1)^{t} \Gamma(k-p-1)^{t}}{\Gamma(k-1)^{t}}\left[G_{\mathbb{F}, k_{1}}, G_{\mathbb{F}, k_{2}}\right]_{\mathbf{p}}^{H i l}$.

Now take $k_{1}=k-q-p-1, k_{2}=q+1-p$ for $q+p \equiv 1(\bmod 2), p, q>0$, to get

$$
\sum_{f \in \mathcal{B}_{k}^{0}}(-1)^{t} \frac{R_{k-q-2}(f) R_{k-p-2}(f)}{D^{k-\frac{1}{2}}(2 i)^{t(k-3)}<f, f>} f(\tau)=\frac{2^{2 t} \Gamma(k-p-1)^{t} \Gamma(p+1)^{t}}{\Gamma(k-1)^{t}}\left[G_{\mathbb{F}, k-1-q-p}, G_{\mathbb{F}, q+1-p}\right]_{\mathbf{p}}^{H i l} .
$$

Since

$$
\sum_{f \in \mathcal{B}_{k}^{0}} R_{f}(X, Y) f(\tau)=\sum_{f \in \mathcal{B}_{k}^{0}}(-1)^{t} \frac{R_{f}^{e v}(X) R_{f}^{e v}(Y)+R_{f}^{e v}(Y) R_{f}^{e v}(X)}{D^{k-\frac{1}{2}}(2 i)^{t(k-3)}<f, f>} f(\tau)
$$

we get

$$
\frac{\left\langle b_{\mathbf{k}}(X, Y ; \cdot), f(\cdot)\right\rangle}{\langle f, f\rangle}=R_{f}(X, Y)
$$

Combining all together with Proposition 3.5 we conclude that

$$
C(X, Y ; \tau ; T)=F_{\tau}(T,-X Y T) F_{\tau}(X T, Y T)
$$

which completes a proof.

\section{Conclusion}

One of the main importance of modular forms in number theory is that spaces of modular forms are generated by those with rational Fourier coefficients. The "period theory" gives another natural rational structure of modular forms. A striking result by Zagier [24] states that this rational information of modular forms can be written as a single product of Kronecker series $F_{\tau}(u, v)$ which is a Jacobi form. The recent results in [2, 21] show that Eisenstein-Kronecker numbers have a rich arithmetic nature, such as a connection with the special Hecke $L$-function over imaginary quadratic fields and Katz' two-variable p-adic Eisenstein measure.

In this paper, we identified the Kronecker series as a "Kuznetsov lifting" of holomorphic Hilbert Eisenstein series over totally real number fields with strict class number 1 . This is the first case to connect Kronecker series to the critical 
values of Hilbert modular $L$-functions over a totally real number field and it seems worthwhile to explore the hidden arithmetic relations more.

On the other hand, in terms of geometric interpretation, a modular form can be regarded as a section of a certain sheaf of differential forms on the open modular curve on a congruence subgroup $\Gamma \subset S L_{2}(\mathbb{Z})$. By noting that the singular cohomology of the open modular curve is given by the group cohomology $H^{*}(\Gamma, W)$ the comparison of de Rham and singular cohomology can give an Eichler-isomorphism. Matsushima and Murakami [15] extended the results to show that the space of automorphic forms on a symmetric space $M$ is isomorphic to $H^{*}(M, S)$ for a certain locally constant sheaf $S$ over $M$. The cohomology of Hilbert surfaces in terms of Hilbert cusp forms has been studied by many researchers including [11, 22]. Relating the critical values of $L$-functions of Hilbert cusp forms and cohomology was first studied by Yoshida [22]. Following the work by Knopp [13] and Kohnen-Zagier [14, which provide us the considerable new lights on Eichler-Shimura isomorphisms such as rational structures of elliptic modular forms, we are able to associate the space of Hilbert modular forms over the totally real number fields to the parabolic cohomology group in terms of the period polynomial by taking antiderivative of Hilbert modular form [5].

\section{REFERENCES}

1. A. Babei, L. Rolen And I.WAgner, The Riemann hypothesis for period polynomials of Hilbert modular forms, Journal of Number Theory, Vol. 218, January 2021, page 44 -61 .

2. K. Bannai and S. Kobayashi, Algebraic theta functions and the p-adic interpolation of Eisenstein-Kronecker numbers, Duke Mathematical Journal, 153 (2010), no. 2, 229 295.

3. G. Bol, Invarianten linearer Differentialgleichungen, Abhandlungen aus dem Mathematischen Seminar der Universität Hamburg 16 (1949), no. 3-4, 1 - 28.

4. J. Brunier, G. van der Geer, G. Harder and D. Zagier, The $1-2-3$ of modular forms, Lectures from the Summer School on Modular Forms and their Applications held in Nordfjordeid, June 2004, Edited by Kristian Ranestad, Universitext. Springer-Verlag, Berlin, 2008.

5. Y. Choie, Parabolic cohomology and Hilbert modular forms, Preprint (2021) . 
6. Y. Choie, H. Kim And O. Richter, Differential operators on Hilbert modular forms, Journal of Number Theory 122 (2007), no. 1, 25-36.

7. Y. Chole And M. LeE, Jacobi-like forms, Pseudodifferential operators, and Quasimodular forms, Springer Monographs in Mathematics (2019).

8. Y. Choie, Y. Park and D. Zagier, Periods of modular forms on $\Gamma_{0}(N)$ and Products of Jacobi Theta functions, Journal of the European Mathematical Society, Vol. 21, Issue 5, 1379 - 1410 (2019).

9. M. EichleR, Eine Verallgemeinerung der Abelschen Integrale, Mathematische Zeitschrift, vol. 67 (1957) pp 267-298.

10. M. Eichler And D. Zagier, The theory of Jacobi forms, Progress in Mathematics, 55, Birkhäuser Boston, Inc., Boston, MA, 1985.

11. G. VAN DER GEeR, Hilbert modular surfaces, Ergebnisse der Mathematik und ihrer Grenzgebiete (3) [Results in Mathematics and Related Areas (3)], 16, Springer-Verlag, Berlin, 1988.

12. M. HARRIS, $L$-functions of $2 \times 2$ unitary groups and factorization of periods of Hilbert modulr forms, Journal of American Mathematical Society 6 (1993), no. 3, 637 - 719.

13. M. Knopp, Some new results on the Eichler cohomology of automorphic forms, Bulletin of the American Mathematical Society, 80 (1974), 607 - 632.

14. W. Kohnen And D. Zagier, Modular forms with rational periods, Modular forms (Durham, 1983), 197-249, Ellis Horwood Series in Mathematics and its Applications: Statistics and Operational Research, Horwood, Chichester, 1984.

15. Y. Matsushima and S. Murakami, On vector bundle valued harmonic forms and automorphic forms on symmetric Riemannian manifolds, Annals of Mathematics (2) 78 (1963), $365-416$.

16. Y. Manin, Periods of cusp forms, and $p$-adic Hecke series, (Russian) Matematicheskii Sbornik (N.S.) 92 (134) (1973), 378 - 401, 503.

17. On the critical values of certain Dirichlet series and the periods of automorphic forms, Inventiones Mathematicae 94 (1988), no. 2, 245 - 305.

18. __ Algebraic relations between critical values of zeta functions and inner products, American Journal of Mathematics, 105 (1983), no. 1, 253 - 285.

19. The special values of the zeta functions associated with Hilbert modular forms, Duke Mathematical Journal, 45 (1978), no. 3, 637- 679.

20. __ Sur les intégrales attachées aux forms automorphes, Journal of the Mathematical Society of Japan 11 (1959), 291 - 311.

21. J. Sprang, Eisenstein-Kronecker series via the Poincaré bundle, Forum of Mathematics, Sigma 7 (2019), No. e34, 59 pp.

22. H. YoshidA, Absolute CM-periods. Mathematical Surveys and Monographs, 106, American Mathematical Society, Providence, RI, 2003. 
PERIODS OF HILBERT MODULAR FORMS, KRONECKER SERIES AND COHOMOLOGY9

23. A. WeIL, Elliptic functions according to Eisenstein and Kronecker, Reprint of the 1976 original, Classics in Mathematics, Springer-Verlag, Berlin, 1999.

24. D. ZAGIER, Periods of modular forms and Jacobi theta functions, Inventiones Mathematicae, 104, 449-265 (1991).

25. —_ The Rankin-Selberg method for automorphic functions which are not of rapid decay, Journal of the Faculty of Science, University of Tokyo, Section IA, Mathematics 28 (1981), no. 3, 415 - 437 (1982).

26. On the values at negative integers of the Zeta-functions of a real quadratic fields, L’Enseignement Mathématique, 22 (1976), no. 1 - 2, 55 - 95.

YoungJu Choie

Department of Mathematics

Pohang University of Science and Technology (POSTECH)

Pohang, Republic of Korea

Email address: yjc@postech.ac.kr 